\title{
A THREE YEAR STUDY FOR THE SPATIAL AND TEMPORAL DISTRIBUTION/VARIATION OF PESTICIDES RESIDUES IN VIOTIKOS KIFISSOS BASIN BEFORE AND AFTER THE APPLICATION OF A LOW INPUT CROP MANAGEMENT SYSTEM
}

$\underline{\text { Angeliki Costa Charalampous }}^{\text {a,c*, }}$, Kyriaki Machera $^{\mathrm{b}}$, George Emmanouel Miliadis ${ }^{\mathrm{a}}$, Michael Andrea Koupparis ${ }^{\mathrm{c}}$

\footnotetext{
${ }^{a}$ National Reference Laboratory for Pesticides Residues, Benaki Phytopathological Institute, 14561 Kifissia, Greece

${ }^{b}$ Laboratory of Toxicological Control of Pesticides, Benaki Phytopathological Institute, 14561 Kifissia, Greece

${ }^{c}$ Laboratory of Analytical Chemistry, Department of Chemistry, University of Athens, Panepistimiopolis, Athens 15771, Greece
} 
Table 1S: Name, pesticide class, mode of action, octanol-water partition coefficient as the log value (pKow) and vapour pressure ( $\mathrm{V} p)$ of the studied analytes

\begin{tabular}{|c|c|c|c|c|}
\hline Name & Pesticide class & Mode of action & pKow & $\mathrm{V} p(\mathrm{mPa})$ \\
\hline acetamiprid & neonicotinoid & insecticide & 0.8 & $1.00 \mathrm{E}-03$ \\
\hline acetochlor & chloroacetamide & herbicide & 4.14 & $6.00 \mathrm{E}+00$ \\
\hline aclonifen & diphenyl ether & herbicide & 4.37 & $1.60 \mathrm{E}-02$ \\
\hline acrinathrin & pyrethrin & insecticide & 5.6 & $4.40 \mathrm{E}-05$ \\
\hline alachlor & chloroacetamide & herbicide & 3.09 & $2.00 \mathrm{E}+00$ \\
\hline aldicarb sulfone & carbamate & insecticide & -0.57 & \\
\hline ametryn & triazine & herbicide & 2.63 & $3.65 \mathrm{E}-01$ \\
\hline atrazine & triazine & herbicide & 2.5 & $1.50 \mathrm{E}-04$ \\
\hline azimsulfuron & sulfonylurea & herbicide & 4.43 & $4.00 \mathrm{E}-06$ \\
\hline azinphos ethyl & organophosphate & insecticide & 3.18 & $3.20 \mathrm{E}-01$ \\
\hline azinphos methyl & organophosphate & insecticide & 2.96 & $5.00 \mathrm{E}-04$ \\
\hline azoxystrobin & strobilurin & fungicide & 2.5 & $1.10 \mathrm{E}-07$ \\
\hline benalaxyl & acylalanine & fungicide & 3.54 & $6.60 \mathrm{E}-01$ \\
\hline benfuracarb & carbamate & insecticide & 4.22 & $1.00 \mathrm{E}-02$ \\
\hline bensulfuron methyl & sulfonylurea & herbicide & -0.99 & $2.80 \mathrm{E}-09$ \\
\hline benzoximate & other & acaricide & 2.4 & $4.50 \mathrm{E}-01$ \\
\hline bifenthrin & pyrethrin & insecticide & 6.6 & $2.40 \mathrm{E}-02$ \\
\hline bitertanol & triazole & fungicide & 4.1 & $2.20 \mathrm{E}-07$ \\
\hline boscalid & carboxamide & fungicide & 2.96 & $7.20 \mathrm{E}-04$ \\
\hline bromophos ethyl & organophosphate & insecticide & 6.13 & - \\
\hline bromopropylate & benzilate & acaricide & 5.4 & $6.80 \mathrm{E}-03$ \\
\hline bromuconazole & triazole & fungicide & 3.24 & $4.00 \mathrm{E}-03$ \\
\hline bupirimate & pyrimidinol & fungicide & 3.9 & $1.00 \mathrm{E}-01$ \\
\hline buprofezin & other & insecticide & 4.3 & $1.25 \mathrm{E}+00$ \\
\hline cadusafos & organophosphate & insecticide & 3.9 & $1.20 \mathrm{E}+02$ \\
\hline carbofuran & carbamate & insecticide & 1.52 & $3.10 \mathrm{E}-02$ \\
\hline chlorbromuron & urea & herbicide & 2.9 & - \\
\hline chlordane alpha & cyclodiene & insecticide & 5.57 & $1.30 \mathrm{E}+00$ \\
\hline chlordane gamma & cyclodiene & insecticide & 5.57 & $1.30 \mathrm{E}+00$ \\
\hline chlorfenapyr & arylpyrrole & insecticide & 4.83 & $1.20 \mathrm{E}-02$ \\
\hline chlorfenvinfos & organophosphate & insecticide & 3.85 & $1.00 \mathrm{E}+00$ \\
\hline chlorobenzilate & organochlorine & acaricide & 4.58 & - \\
\hline chlorothalonil & chloronitrile & fungicide & 2.92 & $7.60 \mathrm{E}-02$ \\
\hline chlorotoluron & urea & herbicide & 2.5 & $5.00 \mathrm{E}-03$ \\
\hline chlorpropham & carbamate & herbicide & 3.79 & $2.40 \mathrm{E}+01$ \\
\hline chlorpyrifos & organophosphate & insecticide & 4.7 & $2.70 \mathrm{E}+00$ \\
\hline chlorpyrifos methyl & organophosphate & insecticide & 4.24 & $3.00 \mathrm{E}+00$ \\
\hline chlorsulfuron & sulfonylurea & herbicide & -0.99 & $3.00 \mathrm{E}-06$ \\
\hline clofentezine & tetrazine & acaricide & 4.1 & $1.30 \mathrm{E}-04$ \\
\hline clothianidin & neonicotinoid & insecticide & 0.7 & $1.30 \mathrm{E}-04$ \\
\hline cyfluthrin & pyrethrin & insecticide & 6 & $9.60 \mathrm{E}-04$ \\
\hline cypermethrin & pyrethrin & insecticide & 6.6 & $2.00 \mathrm{E}-04$ \\
\hline
\end{tabular}




\begin{tabular}{|c|c|c|c|c|}
\hline cyproconazole & triazole & fungicide & 3.1 & $2.60 \mathrm{E}-02$ \\
\hline cyprodinil & anilinopyrimidine & fungicide & 3.9 & $5.10 \mathrm{E}-01$ \\
\hline$o, p^{\prime}-\mathrm{DDD}$ & organochlorine & degradation product & 5.39 & - \\
\hline$p, p^{\prime}-\mathrm{DDD}$ & organochlorine & degradation product & 5.39 & - \\
\hline$o, p^{\prime}-\mathrm{DDE}$ & organochlorine & degradation product & 6.2 & - \\
\hline$p, p^{\prime}-\mathrm{DDE}$ & organochlorine & degradation product & 6.2 & - \\
\hline$o, p^{\prime}-\mathrm{DDT}$ & organochlorine & insecticide & 5.92 & $2.50 \mathrm{E}-02$ \\
\hline$p, p^{\prime}-\mathrm{DDT}$ & organochlorine & insecticide & 5.92 & $2.50 \mathrm{E}-02$ \\
\hline deltamethrin & pyrethrin & insecticide & 4.6 & $1.24 \mathrm{E}-05$ \\
\hline lesethylterbuthylazine & triazine & degradation product & 1.85 & - \\
\hline desmetryn & other & herbicide & 2.38 & $1.33 \mathrm{E}-01$ \\
\hline diazinon & organophosphate & insecticide & 3.3 & $1.20 \mathrm{E}+01$ \\
\hline dicloran & aromatic hydrocarbon & fungicide & 2.8 & $1.60 \mathrm{E}-01$ \\
\hline dicofol & other & acaricide & 4.3 & $5.30 \mathrm{E}-02$ \\
\hline dieldrin & cyclodiene & insecticide & 4.88 & - \\
\hline difenoconazole & triazole & fungicide & 4.4 & $3.30 \mathrm{E}-05$ \\
\hline dimethenamide-P & chloroacetamide & herbicide & 1.89 & $2.51 \mathrm{E}+00$ \\
\hline dimethoate & organophosphate & insecticide & 0.704 & $2.50 \mathrm{E}-01$ \\
\hline dimethomorph & cinnamic acid & fungicide & 2.63 & $9.70 \mathrm{E}-04$ \\
\hline diniconazole & triazole & fungicide & 4.3 & $2.93 \mathrm{E}+00$ \\
\hline dinitramine & dinitroaniline & herbicide & 4.3 & $4.79 \mathrm{E}-01$ \\
\hline disulfoton sulfone & organophosphate & insecticide & 3.95 & - \\
\hline diuron & urea & herbicide & 2.85 & $1.10 \mathrm{E}-03$ \\
\hline dodemorph & morpholine & fungicide & 4.14 & 4.80E-02 \\
\hline alpha- endosulfan & cyclodiene & insecticide & 4.74 & $8.30 \mathrm{E}-01$ \\
\hline beta- endosulfan & cyclodiene & insecticide & 4.79 & $8.30 \mathrm{E}-01$ \\
\hline endosulfan sulfate & cyclodiene & insecticide & 3.18 & - \\
\hline epoxiconazole & triazole & fungicide & 3.44 & $1.00 \mathrm{E}-02$ \\
\hline esfenvalerate & pyrethrin & insecticide & 6.22 & $2.00 \mathrm{E}-04$ \\
\hline ethalfluralin & dinitroaniline & herbicide & 5.11 & $1.17 \mathrm{E}+01$ \\
\hline ethion & organophosphate & insecticide & 4.28 & $2.00 \mathrm{E}-01$ \\
\hline ethofumesate & benzofuran & herbicide & 2.7 & $1.00 \mathrm{E}-01$ \\
\hline ethoprophos & organophosphate & insecticide & 3.59 & $4.65 \mathrm{E}+01$ \\
\hline etoxazole & & acaricide & 5.59 & $2.18 \mathrm{E}-03$ \\
\hline fenamidone & imidazolinone & fungicide & 2.8 & $3.40 \mathrm{E}-04$ \\
\hline fenamiphos & organophosphate & nematicide & 3.3 & $1.20 \mathrm{E}-01$ \\
\hline fenamiphos sulfone & organophosphate & nematicide & & \\
\hline fenarimol & pyrimidine & fungicide & 3.69 & $6.50 \mathrm{E}-02$ \\
\hline fenazaquin & other & acaricide & 5.51 & $3.40 \mathrm{E}-03$ \\
\hline fenbuconazole & triazole & fungicide & 3.23 & $3.40 \mathrm{E}-01$ \\
\hline fenhexamide & hydroxyanilide & fungicide & 3.51 & $4.00 \mathrm{E}-04$ \\
\hline fenitrothion & organophosphate & insecticide & 3.43 & $1.80 \mathrm{E}+01$ \\
\hline fenoxycarb & carbamate & insecticide & 4.07 & 8.67E-04 \\
\hline fenpropathrin & pyrethrin & insecticide & 6 & 7.30E-01 \\
\hline fensulfothion & organophosphate & insecticide & 2.23 & - \\
\hline fensulfothionoxon & organophosphate & degradation product & 0.82 & - \\
\hline
\end{tabular}




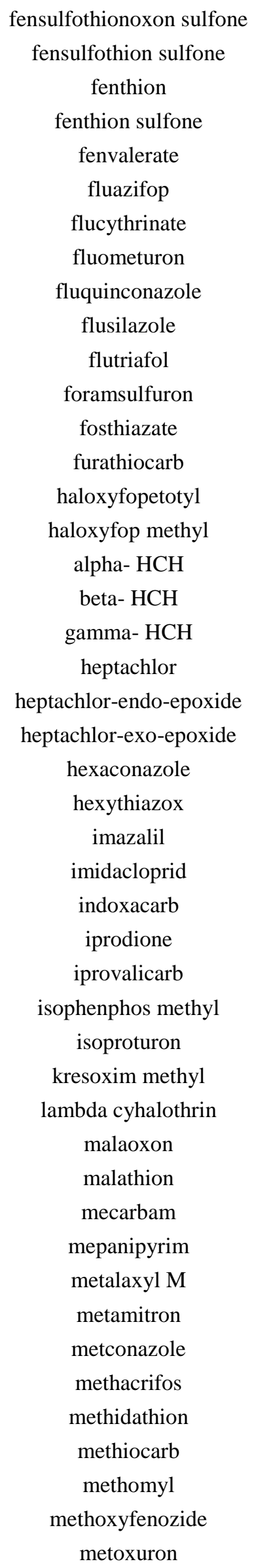

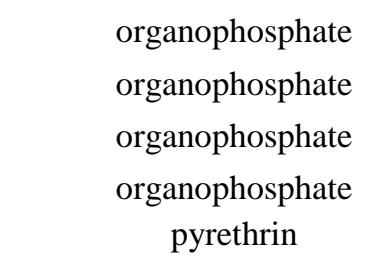

aryloxyphenoxypropionic acid

$$
\text { pyrethrin }
$$$$
\text { urea }
$$

triazole

triazole

triazole

sulfonylurea

organophosphate

carbamate

aryloxyphenoxypropionic ester

aryloxyphenoxypropionic ester organochlorine

organochlorine

organochlorine

organochlorine

organochlorine

organochlorine

$$
\begin{gathered}
\text { triazole } \\
\text { other } \\
\text { imidazole }
\end{gathered}
$$

neonicotinoid

oxadiazine

dicarboximide

carbamate

organophosphate

$$
\text { urea }
$$

oximinoacetate

pyrethrin

organophosphate

organophosphate

organophosphate

anilinopyrimidine

acylalanine

triazinone

triazole

organophosphate

organophosphate

carbamate

carbamate

diacylhydrazine

urea degradation product

degradation product

insecticide

2.43

4.84

7.40E-01

degradation product

1.83

insecticide

5.01

1.92E-02

herbicide

$3.18 \quad 8.00 \mathrm{E}-04$

insecticide

4.7

$1.20 \mathrm{E}-03$

herbicide

2.38

$1.25 \mathrm{E}-01$

fungicide

3.24

$6.40 \mathrm{E}-06$

fungicide

$3.74 \quad 3.90 \mathrm{E}-02$

fungicide

2.3

7.10E-06

herbicide

4.01

4.20E-08

insecticide

1.68

$5.60 \mathrm{E}-01$

insecticide

4.6

$3.90 \mathrm{E}-03$

herbicide

4.33

$1.64 \mathrm{E}-05$

herbicide

insecticide

insecticide

insecticide

insecticide

degradation product

degradation product

fungicide

acaricide

fungicide

insecticide

insecticide

fungicide

fungicide

insecticide

herbicide

fungicide

insecticide

degradation product

4

3.28E-01

3.99

3.99

3.5

4.40E+00

4.95

$5.30 \mathrm{E}+01$

4.24

4.24

3.9

$1.80 \mathrm{E}-02$

$2.53 \quad 3.40 \mathrm{E}-03$

$3.82 \quad 1.58 \mathrm{E}-01$

$0.57 \quad 4.70 \mathrm{E}-07$

$4.65 \quad 2.50 \mathrm{E}-05$

$3 \quad 5.00 \mathrm{E}-04$

$3.2 \quad 4.40 \mathrm{E}-05$

4

2.5

$3.15 \mathrm{E}-03$

3.4

2.30E-03

7

2.00E-04

insecticide

2.07

insecticide

2.75

$5.30 \mathrm{E}+00$

fungicide

2.31

neglible

3.28

2.32E-02

fungicide

1.71

$3.30 \mathrm{E}+00$

herbicide

0.83

8.60E-04

fungicide

3.85

2.10E-05

insecticide

insecticide

2.50E-01

insecticide

2.2

$1.50 \mathrm{E}-02$

insecticide

3.08

$7.20 \mathrm{E}-01$

insecticide

0.093

1.48E-03

herbicide

$4.30 \mathrm{E}+00$ 


\begin{tabular}{|c|c|c|c|c|}
\hline metribuzin & triazinone & herbicide & 1.6 & $5.80 \mathrm{E}-02$ \\
\hline metsulfuron methyl & sulfonylurea & herbicide & 0.018 & 3.30E-07 \\
\hline monocrotophos & organophosphate & insecticide & -0.22 & $2.90 \mathrm{E}-01$ \\
\hline monolinuron & urea & herbicide & 2.2 & $1.30 \mathrm{E}+00$ \\
\hline myclobutanil & triazole & fungicide & 2.94 & $2.13 \mathrm{E}-01$ \\
\hline napropamaide & alkanamide & herbicide & 3.3 & 5.30E-01 \\
\hline nicosulfuron & sulfonylurea & herbicide & -0.36 & 8.00E-07 \\
\hline omethoate & organophosphate & insecticide & -0.74 & $3.30 \mathrm{E}+00$ \\
\hline oxadiazon & oxadiazole & herbicide & 4.91 & $1.00 \mathrm{E}-01$ \\
\hline oxadixyl & oxazolidinone & fungicide & 0.65 & $3.30 \mathrm{E}-03$ \\
\hline oxyfluorfen & diphenyl ether & herbicide & 4.47 & $2.67 \mathrm{E}-02$ \\
\hline paclobutazol & triazole & plant growth regulator & 3.11 & $1.00 \mathrm{E}-03$ \\
\hline parathion & organophosphate & insecticide & 3.83 & $8.90 \mathrm{E}-01$ \\
\hline parathion methyl & organophosphate & insecticide & 3 & $2.00 \mathrm{E}-01$ \\
\hline penconazole & triazole & fungicide & 3.72 & $1.70 \mathrm{E}-01$ \\
\hline pencycuron & phenylurea & fungicide & 4.68 & $5.00 \mathrm{E}-07$ \\
\hline pendimethalin & dinitroaniline & herbicide & 5.2 & $1.94 \mathrm{E}+00$ \\
\hline pentachloroaniline & organochlorine & degradation product & 4.86 & \\
\hline permethrin & pyrethrin & insecticide & 6.1 & $2.00 \mathrm{E}-03$ \\
\hline phenthoate & organophosphate & insecticide & 3.69 & $5.30 \mathrm{E}+00$ \\
\hline phosalone & organophosphate & insecticide & 4.01 & $6.00 \mathrm{E}-02$ \\
\hline phosmet & organophosphate & insecticide & 2.95 & $6.50 \mathrm{E}-02$ \\
\hline phoxim & organophosphate & insecticide & 4.104 & $1.80 \mathrm{E}-01$ \\
\hline pirimicarb & carbamate & insecticide & 1.7 & $4.00 \mathrm{E}-01$ \\
\hline pirimicarb desmethyl & carbamate & degradation product & 0.42 & - \\
\hline pirimiphos methyl & organophosphate & insecticide & 4.2 & $2.00 \mathrm{E}+00$ \\
\hline primisulfuron methyl & sulfonylurea & herbicide & 0.2 & $5.00 \mathrm{E}-03$ \\
\hline prochloraz & imidazole & fungicide & 4.12 & $1.50 \mathrm{E}-01$ \\
\hline profenofos & organophosphate & insecticide & 4.44 & $1.24 \mathrm{E}-01$ \\
\hline prometryn & triazine & herbicide & 4.1 & $1.65 \mathrm{E}-01$ \\
\hline propachlor & chloroacetamide & herbicide & 1.85 & $1.00 \mathrm{E}+01$ \\
\hline propamocarb & carbamate & fungicide & 0.84 & $3.85 \mathrm{E}-02$ \\
\hline propargite & other & acaricide & 5.7 & $4.00 \mathrm{E}-02$ \\
\hline propiconazole & triazole & fungicide & 3.72 & $2.70 \mathrm{E}-02$ \\
\hline propyzamide & benzamide & herbicide & 3.1 & $5.80 \mathrm{E}-02$ \\
\hline prothiofos & organophosphate & insecticide & 5.67 & $3.00 \mathrm{E}-01$ \\
\hline pyraclostrobin & methoxycarbamate & fungicide & 3.99 & $2.60 \mathrm{E}-05$ \\
\hline pyrazophos & phosphorothiolate & fungicide & 3.8 & $2.20 \mathrm{E}-01$ \\
\hline pyridaben & other & insecticide & 6.37 & $1.00 \mathrm{E}-02$ \\
\hline pyrifenox & pyridine & fungicide & 3.4 & $1.70 \mathrm{E}+00$ \\
\hline pyrimethanil & anilinopyrimidine & fungicide & 2.84 & $2.20 \mathrm{E}+00$ \\
\hline pyriproxyfen & other & insecticide & 5.37 & $1.30 \mathrm{E}-02$ \\
\hline quinoxyfen & quinoline & fungicide & 4.66 & $1.20 \mathrm{E}-02$ \\
\hline quizalofop & aryloxyphenoxypropionate acid & herbicide & 4.28 & - \\
\hline quizalofop-P-ethyl & aryloxyphenoxypropionic ester & herbicide & 4.61 & $8.65 \mathrm{E}-04$ \\
\hline resmethrin & pyrethrin & insecticide & 5.43 & - \\
\hline
\end{tabular}




\begin{tabular}{|c|c|c|c|c|}
\hline rimsulfuron & sulfonylurea & herbicide & 0.288 & $1.50 \mathrm{E}-03$ \\
\hline simazine & triazine & herbicide & 2.1 & $2.94 \mathrm{E}-03$ \\
\hline s-metolachlor & chloroacetamide & herbicide & 3.05 & $3.70 \mathrm{E}+00$ \\
\hline sulcotrione & triketone & herbicide & -1.7 & $5.00 \mathrm{E}-03$ \\
\hline tau-fluvalinate & pyrethrin & insecticide & 4.26 & $9.00 \mathrm{E}-08$ \\
\hline tebuconazole & other & fungicide & & - \\
\hline tebufenozide & diacylhydrazine & insecticide & 4.25 & $1.56 \mathrm{E}-04$ \\
\hline tebufenpyrad & other & acaricide & 5.04 & $1.00 \mathrm{E}-02$ \\
\hline tecnazene & aromatic hydrocarbon & fungicide & 4 & $2.40 \mathrm{E}+02$ \\
\hline tefluthrin & pyrethrin & insecticide & 6.4 & $8.40 \mathrm{E}+00$ \\
\hline terbuthylazine & triazine & herbicide & 3.21 & $1.50 \mathrm{E}-01$ \\
\hline tetraconazole & triazole & fungicide & 3.56 & $1.80 \mathrm{E}-01$ \\
\hline tetradifon & other & acaricide & 4.61 & $9.40 \mathrm{E}-07$ \\
\hline thiabentazole & benzimidazole & fungicide & 2.39 & 4.60E-04 \\
\hline thiacloprid & neonicotinoid & insecticide & 1.26 & $3.00 \mathrm{E}-07$ \\
\hline thiamethoxam & neonicotinoid & insecticide & -0.13 & $6.60 \mathrm{E}-06$ \\
\hline thifensulfuron methyl & sulfonylurea & herbicide & 1.06 & $1.70 \mathrm{E}-05$ \\
\hline thiodicarb & oxime carbamate & insecticide & 1.62 & $5.70 \mathrm{E}+00$ \\
\hline tolclofos methyl & aromatic hydrocarbon & fungicide & 4.56 & $5.70 \mathrm{E}+01$ \\
\hline tolyfluanid & sulfamide & fungicide & 3.9 & $2.00 \mathrm{E}-01$ \\
\hline triadimefon & triazole & fungicide & 3.11 & $2.00 \mathrm{E}-02$ \\
\hline triadimenol & triazole & fungicide & 3.08 & $6.00 \mathrm{E}-04$ \\
\hline triazophos & organophosphate & insecticide & 3.34 & $3.90 \mathrm{E}-01$ \\
\hline trifloxystrobin & oximinoacetate & fungicide & 4.5 & $3.40 \mathrm{E}-03$ \\
\hline trifluralin & dinitroaniline & herbicide & 4.83 & $6.10 \mathrm{E}+00$ \\
\hline triticonazole & triazole & fungicide & 3.29 & $1.00 \mathrm{E}-05$ \\
\hline vinclozolin & dicarboximide & fungicide & 3 & $1.30 \mathrm{E}-01$ \\
\hline zoxamide & benzamide & fungicide & 3.76 & $1.00 \mathrm{E}-02$ \\
\hline
\end{tabular}


Table 2S: Recovery results and relative standard deviation values (RSD) for LC amenable pesticides at three different fortification levels

\begin{tabular}{|c|c|c|c|c|c|c|c|}
\hline \multirow[b]{2}{*}{ Analyte } & \multicolumn{2}{|c|}{$\mathrm{C}=0.01 \mu \mathrm{gL}^{-1}$} & \multicolumn{2}{|c|}{$\mathrm{C}=0.1 \mu \mathrm{gL}^{-1}$} & \multicolumn{2}{|c|}{$\mathrm{C}=1 \mu \mathrm{gL}^{-1}$} & \multirow{2}{*}{$\begin{array}{c}\mathrm{LOQ} \\
\left(\mu \mathrm{g} \mathrm{L}^{-1}\right)\end{array}$} \\
\hline & $\begin{array}{c}\text { Recovery } \\
(\%)\end{array}$ & $\begin{array}{c}\text { RSD } \\
(\%)\end{array}$ & $\begin{array}{c}\text { Recovery } \\
(\%)\end{array}$ & $\begin{array}{c}\text { RSD } \\
(\%)\end{array}$ & $\begin{array}{c}\text { Recovery } \\
(\%)\end{array}$ & $\begin{array}{c}\text { RSD } \\
(\%)\end{array}$ & \\
\hline acetamiprid & 111 & 12 & 106 & 4.9 & 119 & 6.8 & 0.01 \\
\hline acetochlor* & - & - & 74.4 & 16.8 & 78 & 11.9 & 0.1 \\
\hline aclonifen & 120 & 15.3 & 135 & 15.1 & 125 & 10.3 & 0.01 \\
\hline alachlor & 92.6 & 10.7 & 98.7 & 4.4 & 124 & 5.2 & 0.01 \\
\hline aldicarb sulfone* & - & - & 127 & 14.1 & 108 & 8.1 & 0.1 \\
\hline ametryn & 119 & 11.8 & 103 & 6.5 & 116 & 9 & 0.01 \\
\hline atrazine & 164 & 14 & 106 & 8 & 99.9 & 7.3 & 0.01 \\
\hline azimsulfuron & 77.4 & 26.7 & 115 & 4.7 & 115.9 & 9.8 & 0.01 \\
\hline azinphos ethyl & 93.9 & 22.7 & 10.5 & 10.7 & 115 & 9.8 & 0.01 \\
\hline azinphos methyl & 102 & 27.6 & 105 & 17.3 & 114 & 8.7 & 0.01 \\
\hline azoxystrobin & 96.3 & 5.9 & 98.7 & 2.8 & 117 & 6.2 & 0.01 \\
\hline benalaxyl & 98 & 12.2 & 103 & 3.1 & 112 & 7 & 0.01 \\
\hline bensulfuron methyl & 119 & 13.2 & 99.2 & 7.6 & 141 & 10.7 & 0.01 \\
\hline benzoxymate & 82.3 & 16.5 & 119 & 9 & 111 & 9.7 & 0.01 \\
\hline bitertanol & 110.4 & 13 & 111 & 8.6 & 107 & 8.4 & 0.01 \\
\hline bromophos ethyl & 94 & 16.5 & 78.5 & 12.5 & 64.2 & 20.1 & 0.01 \\
\hline bromuconazole & 118 & 22.7 & 99.8 & 10.8 & 96.1 & 9.4 & 0.01 \\
\hline bupirimate & 131 & 10.2 & 106 & 5.9 & 109.7 & 7.5 & 0.01 \\
\hline buprofezin & 104 & 16.6 & 105 & 9.5 & 110 & 12.9 & 0.01 \\
\hline caduzafos & 82.1 & 10.1 & 80 & 26.7 & 91.4 & 6.8 & 0.01 \\
\hline carbofuran & 140 & 8.3 & 128.2 & 8.1 & 114 & 4.9 & 0.01 \\
\hline chlorfenvinfos & 91.6 & 6.2 & 107 & 6.9 & 111 & 6.2 & 0.01 \\
\hline chlorobromuron & 99 & 13.8 & 123 & 15.4 & 109 & 7.7 & 0.01 \\
\hline chlorpyrifos ethyl & 94.5 & 10.6 & 85.6 & 10.4 & 76.4 & 11.9 & 0.01 \\
\hline chlorsulfuron & 89.8 & 11.4 & 99 & 10.3 & 103 & 9.2 & 0.01 \\
\hline chlortoluron & 124 & 14.3 & 127 & 14.8 & 123 & 9.2 & 0.01 \\
\hline clofentezine & 75.3 & 13.4 & 91.3 & 7.5 & 72 & 5.9 & 0.01 \\
\hline clothianidin & 106 & 10.6 & 109.3 & 11 & 116 & 7.1 & 0.01 \\
\hline cyproconazole & 94.9 & 19.9 & 118 & 9.4 & 118 & 5.6 & 0.01 \\
\hline cyprodinil & 112 & 6.5 & 110 & 6.3 & 110 & 6.7 & 0.01 \\
\hline desethylterbuthylazine & 75.3 & 14.3 & 91.9 & 12.5 & 91.7 & 2.45 & 0.01 \\
\hline desmetryn & 105 & 14.5 & 88.1 & 6.2 & 114 & 6.9 & 0.01 \\
\hline diazinon & 94.6 & 8.5 & 80.7 & 17.4 & 102 & 5.5 & 0.01 \\
\hline difenoconazole & 110 & 14.4 & 99.1 & 4.7 & 99.1 & 8.1 & 0.01 \\
\hline dimethenamide-P & 78.4 & 12.2 & 80.5 & 15 & 70 & 17.3 & 0.01 \\
\hline dimethoate & 68.2 & 8.3 & 92.6 & 4.5 & 118.5 & 6.4 & 0.01 \\
\hline
\end{tabular}




\begin{tabular}{|c|c|c|c|c|c|c|c|}
\hline dimethomorph & 105 & 12.7 & 104 & 4.4 & 116 & 7.2 & 0.01 \\
\hline diniconazole & 101 & 12.1 & 113 & 6.8 & 108 & 7 & 0.01 \\
\hline disulfoton sulfone & 163 & 8.6 & 129 & 12.7 & 121 & 6.7 & 0.01 \\
\hline epoxiconazole & 84.6 & 14 & 97.4 & 7.1 & 108.3 & 4.5 & 0.01 \\
\hline ethion & 77.4 & 19.2 & 81.9 & 3.5 & 72.8 & 14.8 & 0.01 \\
\hline ethofumesate & 125 & 11.6 & 109 & 8.1 & 124 & 7 & 0.01 \\
\hline ethoprophos & 83.4 & 9.4 & 79.2 & 32 & 98.7 & 8.5 & 0.01 \\
\hline etoxazole & 72.4 & 18.7 & 66.9 & 9.9 & 68.8 & 17 & 0.01 \\
\hline fenamidone* & - & - & 104 & 16.4 & 115 & 6.3 & 0.1 \\
\hline fenamiphos* & - & - & 114 & 5.8 & 97.3 & 13.1 & 0.1 \\
\hline fenamiphos sulfone* & - & - & 126 & 15 & 119 & 6.4 & 0.1 \\
\hline fenarimol & 116 & 19.6 & 73.8 & 10.8 & 121 & 7.8 & 0.01 \\
\hline fenazaquin & 66.7 & 17.3 & 112 & 12.3 & 64.1 & 18.8 & 0.01 \\
\hline fenbuconazole* & - & - & 95.7 & 18 & 113 & 5.8 & 0.1 \\
\hline fenhexamide* & - & - & 131 & 20.2 & 116 & 4 & 0.1 \\
\hline fenitrothion* & - & - & 70.1 & 23.9 & 77.4 & 11 & 0.1 \\
\hline fenoxycarb* & - & - & 79 & 10.1 & 116 & 4.4 & 0.1 \\
\hline fenpropymorph* & - & - & 68.6 & 21.6 & 102 & 6.5 & 0.1 \\
\hline fenpyroximate & 61.8 & 13.4 & 76.9 & 10.8 & 60.8 & 17.8 & 0.01 \\
\hline fensulfothion & 94.4 & 24.2 & 143 & 8.5 & 120 & 11.6 & 0.01 \\
\hline fensulfothionoxon & 106 & 23.9 & 114 & 5.7 & 115 & 6.3 & 0.01 \\
\hline fensulfothionoxon sulfone & 94.8 & 22.3 & 144 & 9.3 & 121 & 12 & 0.01 \\
\hline fensulfothion sulfone* & - & - & 113 & 18.3 & 107 & 13 & 0.1 \\
\hline fenthion* & - & - & 81.2 & 23.5 & 102 & 8.6 & 0.1 \\
\hline fenthion sulfone & 127 & 13.6 & 100 & 15.1 & 119 & 7.2 & 0.01 \\
\hline fluazifop & 51.9 & 15.9 & 51.8 & 19.3 & 52.1 & 9.46 & 0.01 \\
\hline flucythrinate & 88.3 & 10.5 & 118 & 17.2 & 119 & 9 & 0.01 \\
\hline fluquinconazole* & - & - & 137 & 22.6 & 120 & 8.6 & 0.1 \\
\hline flusilazole & 133 & 11.1 & 118 & 5.4 & 109 & 6 & 0.01 \\
\hline foramsulfuron & 69.1 & 19.7 & 72 & 18.4 & 81.7 & 3.28 & 0.01 \\
\hline fosthiazate & 99.9 & 8.4 & 109 & 5.2 & 120 & 6.4 & 0.01 \\
\hline furathiocarb & & & 109 & 14.2 & 87.7 & 12.8 & 0.01 \\
\hline haloxyfop ethyl ester & 99.2 & 15 & 73.1 & 14.2 & 87.1 & 14 & 0.01 \\
\hline $\begin{array}{l}\text { haloxyfopmethoxyethyl } \\
\text { ester }\end{array}$ & 96.6 & 21.4 & 91.7 & 5.9 & 99.3 & 7.5 & 0.01 \\
\hline hexaconazole & 116 & 16 & 102 & 7.5 & 105 & 7 & 0.01 \\
\hline hexythiazox & 101 & 11.8 & 94.1 & 5.7 & 91.1 & 11 & 0.01 \\
\hline imazalil & 98.9 & 7.1 & 86 & 9.1 & 106 & 7.2 & 0.01 \\
\hline imidacloprid & 119 & 20.2 & 110 & 14.7 & 113 & 10.7 & 0.01 \\
\hline iprovalicarb & 110 & 13.8 & 108 & 5.1 & 115 & 6 & 0.01 \\
\hline isophenphos methyl & 80.7 & 9.1 & 74.8 & 14.3 & 102 & 6.5 & 0.01 \\
\hline isoproturon* & - & - & 107 & 12.1 & 119 & 5.8 & 0.1 \\
\hline
\end{tabular}




\begin{tabular}{|c|c|c|c|c|c|c|c|}
\hline kresoxim methyl & 110 & 19.3 & 94.5 & 13.8 & 115 & 7.6 & 0.01 \\
\hline linuron & 127 & 12.8 & 131 & 17.4 & 125 & 18.5 & 0.01 \\
\hline malaoxon & 110 & 11.1 & 99.4 & 5.9 & 117 & 5.2 & 0.01 \\
\hline malathion* & - & - & 118 & 10.3 & 130 & 10.2 & 0.1 \\
\hline mecarbam & 92.9 & 21.7 & 91.7 & 10.6 & 119 & 7.6 & 0.01 \\
\hline mepanipyrim & 106 & 15.7 & 110 & 6.5 & 103 & 5.6 & 0.01 \\
\hline metalaxyl M & 112 & 5.7 & 112 & 7.4 & 122 & 7.1 & 0.01 \\
\hline metamitron & 72.5 & 19.3 & 90 & 10.9 & 108 & 7.6 & 0.01 \\
\hline metconazole & 96.8 & 24.5 & 103 & 10.1 & 114 & 6.9 & 0.01 \\
\hline methidathion* & - & - & 85.9 & 21.2 & 99.8 & 7.9 & 0.1 \\
\hline methiocarb* & - & - & 132 & 22.1 & 135 & 7.8 & 0.01 \\
\hline methomyl & 83.5 & 17 & 59.9 & 10.8 & 107 & 7.4 & 0.01 \\
\hline methoxychlor* & - & - & 87.8 & 9 & 92.4 & 13.7 & 0.1 \\
\hline methoxyfenozide & 106 & 8.1 & 110.8 & 2.1 & 121.8 & 6.3 & 0.01 \\
\hline metoxuron & 110 & 19.2 & 116 & 5.7 & 120 & 5.7 & 0.01 \\
\hline metribuzin* & - & - & 83.1 & 23 & 107 & 12.8 & 0.1 \\
\hline metsulfuron methyl & 118 & 10.9 & 127 & 5.1 & 135 & 6.2 & 0.01 \\
\hline monocrotophos* & - & - & 132 & 4.3 & 121 & 9 & 0.1 \\
\hline monolinuron & 115 & 25.1 & 93 & 16.2 & 117 & 5.6 & 0.01 \\
\hline myclobutanil* & - & - & 130 & 19.1 & 94.5 & 12.1 & 0.1 \\
\hline napropamide & 76.8 & 14.1 & 83.9 & 15 & 88.8 & 6.42 & 0.01 \\
\hline nicosulfuron & 93.7 & 11.4 & 94.3 & 6.9 & 114 & 5.1 & 0.01 \\
\hline oxadiazon & 110 & 12.6 & 85.9 & 1.7 & 97.6 & 9.1 & 0.01 \\
\hline oxadixyl & 144 & 22.8 & 136 & 14.1 & 116 & 11.8 & 0.01 \\
\hline paclobutazole & 89.9 & 11.3 & 105 & 9 & 117 & 5.2 & 0.01 \\
\hline penconazole & 84.1 & 12.8 & 111 & 7.3 & 106 & 5.8 & 0.01 \\
\hline pencycuron* & - & - & 124 & 4.6 & 115 & 6.8 & 0.1 \\
\hline pendimethalin & 94.9 & 6.7 & 85.3 & 6.3 & 74.5 & 16 & 0.01 \\
\hline phosalone & 109 & 23.2 & 115 & 14.2 & 104 & 8.3 & 0.01 \\
\hline phosmet & 66 & 19 & 119 & 21.7 & 125 & 5.4 & 0.01 \\
\hline phoxim* & - & - & 101 & 23.2 & 109 & 8.5 & 0.1 \\
\hline pirimicarb & 104 & 8.7 & 125 & 6 & 122 & 5 & 0.01 \\
\hline pirimicarb desmethyl & 120 & 6.8 & 130 & 7.9 & 120 & 6.2 & 0.01 \\
\hline pirimiphos methyl & 115 & 5.6 & 99.4 & 10.6 & 100 & 11.7 & 0.01 \\
\hline primisulfuron methyl & 71.4 & 23.7 & 118 & 19.8 & 119 & 8.9 & 0.01 \\
\hline procloraz* & - & - & 109 & 10.7 & 106 & 6.4 & 0.1 \\
\hline profenofos & 132 & 12.2 & 103 & 13.4 & 83.3 & 16.5 & 0.01 \\
\hline prometryn & 101 & 5.9 & 93.1 & 3.2 & 114 & 7.3 & 0.01 \\
\hline propamocarb* & - & - & 73.1 & 22.4 & 98.8 & 7.2 & 0.1 \\
\hline propargite & 92.4 & 12.6 & 79.4 & 8.9 & 70.7 & 18.9 & 0.01 \\
\hline propyconazole & 115 & 13.9 & 97 & 5.6 & 103 & 7.1 & 0.01 \\
\hline pyraclostrobin & 102 & 5.5 & 100 & 10.9 & 96.5 & 10.1 & 0.01 \\
\hline
\end{tabular}




\begin{tabular}{cccccccc} 
pyrazophos & 87.2 & 13.6 & 101 & 14.6 & 101 & 11.4 & 0.01 \\
pyrifenox & 104 & 11.9 & 100 & 8.7 & 111 & 6.1 & 0.01 \\
pyrimethanil & 105 & 16.1 & 115 & 10.1 & 115 & 6.1 & 0.01 \\
pyriproxyfen & 80.6 & 9 & 76.2 & 9 & 71.6 & 14.9 & 0.01 \\
quiazalofop & 88.5 & 26.6 & 93.7 & 20.3 & 101 & 11.6 & 0.01 \\
quinoxyfen & 110 & 11.9 & 81.9 & 12.8 & 77.7 & 14.9 & 0.01 \\
rimsulfuron & 62.3 & 15.3 & 71.1 & 19.9 & 89 & 10.4 & 0.01 \\
s-metolachlor & 71.5 & 21.3 & 86.8 & 12.1 & 95.7 & 6.24 & 0.01 \\
simazine & 94.9 & 20.5 & 110 & 12.3 & 120 & 8.2 & 0.01 \\
tebuconazole & 84.3 & 11.8 & 99 & 8.1 & 123 & 9.9 & 0.01 \\
tebufenozide & 112 & 8 & 96 & 10.9 & 115 & 7.6 & 0.01 \\
tebufenpyrad* & - & - & 112 & 11.8 & 82.4 & 13.4 & 0.1 \\
terbuthylazine & 98.9 & 6.5 & 98.4 & 1.8 & 120 & 7.5 & 0.01 \\
tetraconazole & 100 & 6.6 & 100 & 9.2 & 109 & 7.2 & 0.01 \\
thiabentazole & 60.5 & 12.2 & 56 & 9.9 & 116 & 7.2 & 0.01 \\
thiacloprid & 112 & 6.6 & 92.6 & 6 & 112 & 11.9 & 0.01 \\
thiamethoxam & 104 & 15.1 & 110 & 3.4 & 124 & 3.5 & 0.01 \\
thifensulfuron methyl & 111 & 4.4 & 88.4 & 6.2 & 118 & 10.8 & 0.01 \\
thiodicarb & 113 & 19.6 & 113 & 4.3 & 124 & 5.1 & 0.01 \\
toclofos methyl & 99.2 & 12 & 99.6 & 22.4 & 94 & 8.2 & 0.01 \\
tolyfluanid & 66.4 & 14.1 & 54.8 & 29.9 & 123 & 5.8 & 0.01 \\
triadimenol & 87.9 & 31.2 & 109 & 16.2 & 127 & 6.3 & 0.01 \\
triazophos & 110 & 6.1 & 96.3 & 5.7 & 114 & 6.9 & 0.01 \\
trifloxystrobin & 102 & 9.8 & 80.5 & 4 & 94.6 & 11.5 & 0.01 \\
triticonazole & 112 & 16 & 99.6 & 6.1 & 133 & 6.3 & 0.01 \\
zoxamide & 112 & 2.8 & 98.5 & 4.4 & 112 & 4.8 & 0.01 \\
\hline
\end{tabular}

*For these analytes signal to noise ratio for quantification ion transition at the lowest fortification level was $<10$. 
Table 3S: Recovery results with relative standard deviation values (RSD) for two different fortification levels and limit of quantitation (LOQ) for GC amenable pesticides.

\begin{tabular}{|c|c|c|c|c|c|}
\hline \multirow{2}{*}{ Analyte } & \multicolumn{2}{|c|}{$\mathrm{C}=0.01 \mu \mathrm{gL}^{-1}$} & \multicolumn{2}{|c|}{$\mathrm{C}=0.1 \mu \mathrm{gL}^{-1}$} & \multirow{2}{*}{$\begin{array}{l}\text { LOQ } \\
\left(\mu g L^{-1)}\right.\end{array}$} \\
\hline & $\begin{array}{c}\text { Recovery } \\
(\%)\end{array}$ & $\begin{array}{c}\text { RSD } \\
(\%)\end{array}$ & $\begin{array}{c}\text { Recovery } \\
(\%)\end{array}$ & $\begin{array}{c}\text { RSD } \\
(\%)\end{array}$ & \\
\hline acrinathrin & 34.8 & 25.1 & 31.5 & 12.1 & 0.01 \\
\hline benalaxyl & 87.2 & 21 & 85.6 & 9 & 0.01 \\
\hline bifenthrin & 58.7 & 25.2 & 67 & 11.6 & 0.01 \\
\hline bitertanol & 93.9 & 14.1 & 114.6 & 15.3 & 0.01 \\
\hline bromophos ethyl & 78.9 & 31.3 & 89.9 & 18.7 & 0.01 \\
\hline bromopropylate & 105.5 & 19.8 & 105 & 23.5 & 0.01 \\
\hline chlorpyrifos methyl & 99.7 & 10.8 & 84.8 & 6.7 & 0.01 \\
\hline chlordane alpha & 65.2 & 9.5 & 63.5 & 12.7 & 0.01 \\
\hline chlordane gamma & 100.7 & 20.7 & 95.5 & 19.5 & 0.01 \\
\hline chlorfenapyr & 71.2 & 14.9 & 133 & 8 & 0.01 \\
\hline chlorobenzilate & 113 & 20.7 & 80.1 & 6 & 0.01 \\
\hline chlorothalonil & 124 & 22.3 & 88.8 & 14 & 0.01 \\
\hline chlorpropham & 134.4 & 26.3 & 92.4 & 5.1 & 0.01 \\
\hline chlorpyrifos & 102 & 10.8 & 111 & 11.2 & 0.01 \\
\hline cyfluthrin & 45 & 6 & 64.6 & 7.5 & 0.01 \\
\hline cypermethrin & 94.5 & 14.8 & 68.4 & 7.2 & 0.01 \\
\hline$o, p^{\prime}-\mathrm{DDD}$ & 81 & 21.2 & 94.9 & 14.8 & 0.01 \\
\hline$p, p^{\prime}-\mathrm{DDD}$ & 81.1 & 31.8 & 90.2 & 16 & 0.01 \\
\hline$o, p^{\prime}-\mathrm{DDE}$ & 74.2 & 20.6 & 65.4 & 15.5 & 0.01 \\
\hline$p, p^{\prime}-\mathrm{DDE}$ & 75.1 & 23.1 & 80.4 & 13.9 & 0.01 \\
\hline$o, p^{\prime}-\mathrm{DDT}$ & 77.3 & 32.3 & 89.2 & 16.3 & 0.01 \\
\hline$p, p^{\prime}-\mathrm{DDT}$ & 90.8 & 14.5 & 105 & 11.5 & 0.01 \\
\hline deltamethrin & 56.3 & 7.7 & 62.9 & 16.7 & 0.01 \\
\hline desmetryn & 96.5 & 10.4 & 88.4 & 8.4 & 0.01 \\
\hline diazinon & 107 & 18.5 & 93.4 & 10.2 & 0.01 \\
\hline dicloran & 82.2 & 26.9 & 78.6 & 4.8 & 0.01 \\
\hline$p, p^{\prime}-$ dicofol & 91.1 & 24.6 & 111 & 7.8 & 0.01 \\
\hline dieldrin & 101 & 16 & 86.2 & 12.8 & 0.01 \\
\hline dinitramine & 78.1 & 17.4 & 89.6 & 18.7 & 0.01 \\
\hline alpha - endosulfan & 73.7 & 7.6 & 78.3 & 14 & 0.01 \\
\hline beta - endosulfan & 121 & 21.6 & 74.5 & 14.3 & 0.01 \\
\hline endosulfan sulfate & 82.1 & 7.6 & 87.8 & 6.6 & 0.01 \\
\hline esfenvalerate & 46.7 & 16.2 & 54.7 & 22.1 & 0.01 \\
\hline ethalfluralin & 62.2 & 18.5 & 80.7 & 14 & 0.01 \\
\hline ethion & 68.5 & 20.7 & 113 & 16.9 & 0.01 \\
\hline fenamiphos & 61.3 & 23.6 & 90.1 & 13.4 & 0.01 \\
\hline fenpropathrin & 56 & 12.5 & 76 & 18.8 & 0.01 \\
\hline fenvalerate & 46.2 & 12.6 & 66.6 & 14.8 & 0.01 \\
\hline flucythrinate & 57.6 & 7.12 & 66.3 & 10.5 & 0.01 \\
\hline alpha - HCH & 88.5 & 15.9 & 103 & 13.1 & 0.01 \\
\hline
\end{tabular}




\begin{tabular}{cccccc} 
beta - HCH & 79.3 & 11.6 & 89.2 & 4.7 & 0.01 \\
gamma - HCH & 111 & 18.01 & 99.6 & 6.1 & 0.01 \\
heptachlor-endo-epoxide & 108 & 18.5 & 92.3 & 10.6 & 0.01 \\
heptachlor-exo-epoxide & 95.2 & 21.9 & 87 & 7.5 & 0.01 \\
heptachlor & 89.3 & 20.6 & 65.6 & 11.6 & 0.01 \\
imazalil & 114 & 17.2 & 78 & 10.7 & 0.01 \\
iprodione & 95.4 & 20.8 & 81.3 & 19.1 & 0.01 \\
lambda cyhalothrin & 44.5 & 10 & 48.6 & 9.4 & 0.01 \\
pentachloroaniline & 98.6 & 22.1 & 107 & 9.1 & 0.01 \\
permethrin & 66.02 & 18.8 & 64.7 & 21.4 & 0.01 \\
phenthoate & 122 & 14.5 & 98.6 & 4.2 & 0.01 \\
pirimiphos methyl & 107 & 9.2 & 97.6 & 6.8 & 0.01 \\
profenofos & 90.1 & 14.6 & 118 & 17.1 & 0.01 \\
prometryn & 103 & 18.1 & 97.7 & 5.6 & 0.01 \\
propachlor* & - & - & 92.4 & 14.4 & 0.1 \\
propyzamide & 110 & 13.8 & 104 & 5 & 0.01 \\
prothiofos & 91.3 & 32.7 & 87.2 & 19.1 & 0.01 \\
resmethrin* & - & - & 90.8 & 18.8 & 0.1 \\
tau-fluvalinate* & - & - & 62.3 & 23.5 & 0.1 \\
tecnazene & 79.5 & 19.6 & 68.2 & 10.5 & 0.01 \\
tefluthrin & 81.2 & 19.2 & 63.7 & 13.4 & 0.01 \\
tetradifon & 93.1 & 33.2 & 114 & 18.3 & 0.01 \\
tolclofos methyl & 94.3 & 21.2 & 85.3 & 5.4 & 0.01 \\
trifluralin & 76.3 & 20.1 & 86.5 & 16.1 & 0.01 \\
vinclozolin & 128 & 17.9 & 89.6 & 7.3 & 0.01 \\
\hline
\end{tabular}

*For these analytes signal to noise ratio for quantification ion transition at lowest fortification level was $<10$. 